\title{
Reactions to abortion and subsequent mental health
}

\author{
David M. Fergusson, L. John Horwood and Joseph M. Boden
}

\section{Background}

There has been continued interest in the extent to which women have positive and negative reactions to abortion.

\section{Aims \\ To document emotional reactions to abortion, and to examine the links between reactions to abortion and subsequent mental health outcomes.}

\section{Method}

Data were gathered on the pregnancy and mental health history of a birth cohort of over 500 women studied to the age of 30 .

\section{Results}

Abortion was associated with high rates of both positive and negative emotional reactions; however, nearly $90 \%$ of respondents believed that the abortion was the right decision. Analyses showed that the number of negative responses to the abortion was associated with increased levels of subsequent mental health disorders $(P<0.05)$. Further analyses suggested that, after adjustment for confounding, those having an abortion and reporting negative reactions had rates of mental health disorders that were approximately 1.4-1.8 times higher than those not having an abortion.

\section{Conclusions}

Abortion was associated with both positive and negative emotional reactions. The extent of negative emotional reactions appeared to modify the links between abortion and subsequent mental health problems.

\section{Declaration of interest}

None.
In a previous paper ${ }^{1}$ we examined the links between the outcomes of pregnancy (including pregnancy loss, abortion and unwanted pregnancy coming to term) and mental health in a birth cohort of New Zealand women studied to the age of 30. That analysis showed that although exposure to abortion was associated with a modest increase in the risks of mental health problems (relative risk $(\mathrm{RR})=1.3$ approximately), there was no consistent association between other pregnancy outcomes and mental health. These findings persisted after extensive control for prospective and concurrent covariates and were found for a range of models using varying lag assumptions. These findings clearly suggested that unwanted pregnancy leading to abortion was likely to be a risk factor for subsequent mental health problems, whereas unwanted pregnancy leading to live birth was not a risk factor for these problems.

Recently there have been two major US-based reviews of the evidence on the links between abortion and mental health. Both of these reviews have concluded that evidence from better-quality studies does not support the view that abortion is associated with increased risks of mental health problems. ${ }^{2,3}$ However, both reviews contained a major flaw that has limited both the generality and utility of their conclusions. This flaw centres around a failure to distinguish between two closely related questions about the causal effects of abortion on mental health. The first question focuses on whether unwanted pregnancy terminated by abortion is an adverse life event that leads to increased risks of mental health problems in women exposed to that event. Addressing this question requires comparing women exposed to abortion with an equivalent group of women not exposed to abortion. Answering this question is important for understanding the extent to which the termination of unwanted pregnancy may be associated with adverse mental health consequences. The second causal question concerns the issue of whether any adverse consequences of unwanted pregnancy terminated by abortion are greater or lesser than the adverse consequences of unwanted pregnancy coming to term. Addressing this question requires comparing the outcomes of women having abortions with the outcomes of an equivalent group of women coming to term with unwanted pregnancies. Answering this question is important for understanding the extent to which abortion may exacerbate or mitigate any mental health risks associated with unwanted pregnancy. Both US reviews committed the error of dismissing large amounts of evidence that was relevant to answering the first question on the grounds that these data failed to address the second question.

The present paper reports an extension of our previous study. ${ }^{1}$ Here we view unwanted pregnancy terminated by abortion as an adverse life event that is potentially associated with increased risks of mental health problems. From this perspective, an important question concerns the extent to which the woman's reactions to abortion may modify risks of mental health problems. In particular, it would be expected that if unwanted pregnancy terminated by abortion is an adverse life event that provokes mental health problems, this association would be influenced by the extent to which the women perceived the abortion as causing distress, threat or guilt. More specifically, it would be hypothesised that any adverse mental health effects of unwanted pregnancy terminated by abortion will be confined to those women who report significant distress about the abortion.

There have been a number of studies that have examined women's reactions to abortion. In general, these studies have found that women report relatively high rates of both positive and negative reactions to abortion. ${ }^{4-14}$ For example, Kero et al found that over $80 \%$ of women reported at least one negative response, with just over two-thirds reporting a positive response. ${ }^{7}$ The majority $(52 \%)$ of respondents reported a mixture of positive and negative responses. These findings clearly suggest considerable variation in the reactions of women to abortion.

Although responses to abortion have been well documented, few studies have examined the links between reactions to abortion and subsequent mental health. However, Major and colleagues, using a sample of over 800 women who had undergone an abortion, found correlations ranging from 0.25 to 0.40 between 
participants' extent of abortion-related distress and subsequent mental health problems. ${ }^{4}$ Against this background, the present paper reports on: the frequency of positive and negative reactions to abortion assessed by retrospective reports gathered at age 30; the relationships between reported reactions to abortion and subsequent mental health; and a re-analysis of our previous data to examine the extent to which reactions to abortion modified the links between abortion and later mental health disorders.

\section{Method}

The data used in this analysis were gathered over the course of the Christchurch Health and Development Study (CHDS). The CHDS is a longitudinal study of a birth cohort of 1265 children born in the Christchurch (New Zealand) urban region who have been studied at birth, 4 months, 1 year and at annual intervals to age 16 years, and again at ages 18,21, 25 and 30 . The present analysis is based on the cohort of female participants for whom information on pregnancy history and mental health outcomes was available. All data were collected only on the basis of signed consent from research participants. The study had ethical approval from the Canterbury Ethics Committee.

\section{Pregnancy and abortion history}

At each assessment from age 15 to 30 years, participants were questioned about any pregnancies occurring since the previous assessment, and the timing and outcome of each reported pregnancy was recorded. In addition, at age 30 only, participants were asked to provide a summary of their full pregnancy history. This summary provided information on pregnancy timing, number of pregnancies and pregnancy outcomes (as a check on prospective data). A further aspect of the age 30 pregnancy history summary was that participants were also questioned about whether each pregnancy was wanted or unwanted, and their initial reaction to the pregnancy at the time. Initial reactions were coded on a five-point scale from very happy to very unhappy/distressed.

As described in a previous paper, ${ }^{1}$ the combined retrospective and prospective report data were used to define a series of summary measures reflecting the woman's pregnancy history for any given period of interest. Specifically, the information on timing and outcome for each reported pregnancy was used to classify women on four measures of pregnancy outcome in any given interval: whether the woman reported having an elective abortion during the interval; whether the woman experienced a pregnancy loss (miscarriage, stillbirth, and termination of ectopic pregnancy) during the interval; whether the woman had a live birth during the interval for which she reported an unwanted or adverse reaction to the pregnancy; and whether the woman had a live birth during the interval with no adverse reaction to the pregnancy. Based on the combined report data, 284 women (53\% of the cohort) reported a total of 686 pregnancies before age 30 including: 153 abortions (occurring to 117 women), 138 pregnancy losses (to 95 women), 66 live births (to 52 women) following an unwanted/adverse reaction to the pregnancy, and 329 other live births (to 197 women).

Of the 117 women who reported an abortion, 79 (68\%) provided consistent prospective and retrospective reports; 25 (21\%) reported an abortion retrospectively but not prospectively; and $13(11 \%)$ reported an abortion prospectively but not retrospectively. The overall kappa between prospective and retrospective reports of abortion was 0.76, suggesting an acceptable level of reporting reliability. Comparison of the mental heath histories of those consistently reporting abortion with those giving inconsistent reports showed no significant differences in the overall rates of mental health problems in these groups.

\section{Reactions to abortion}

As part of the assessment at age 30 years, women who had had an induced abortion were questioned about their reactions to abortion. Women who had experienced more than one abortion were asked to respond about their reactions to their first abortion. Participants were asked about the extent to which they experienced any of a series of nine emotional responses immediately following their abortion. These responses included both positive (relief, happiness, satisfaction) and negative (sorrow, sadness, guilt, regret, grief/loss, disappointment) responses. Questioning was based on the measures used by Major et $a l^{4}$ and Broen et al. ${ }^{5}$ Items were coded on a three-point scale reflecting the extent of response (not at all, somewhat, very much). Participants were also asked how they felt in hindsight about their decision to have a termination, again based on the measure used by Major et al. ${ }^{4}$ Responses were made on a threepoint scale: definitely the wrong decision; not sure; definitely the right decision.

\section{Mental health}

At each assessment from age 16 to 30 years, participants were questioned about mental health issues since the previous assessment using structured questionnaires based on the Diagnostic Interview Schedule for Children (DISC) ${ }^{15}$ at age 16 years and the Composite International Diagnostic Interview $(\mathrm{CIDI})^{16}$ at ages $18-30$ years, supplemented by additional measures. From this questioning it was possible to ascertain the proportion of young women who met DSM-IV ${ }^{17}$ diagnostic criteria for the following disorders during the intervals 15-18, $18-21,21-25$ and $25-30$ years: major depression; anxiety disorders (including generalised anxiety, panic disorder, agoraphobia, social phobia and specific phobia); alcohol dependence; and illicit drug dependence. In addition, measures of DSM-IV disorders were supplemented by measures of self-reported suicidal ideation. To provide an overall measure of the burden of mental disorder, the five individual measures were summed to obtain a count of the number of mental health problems reported for each interval. This count measure was the primary outcome used in the present analysis.

\section{Covariate factors}

A wide range of factors were considered for inclusion as covariates in the analysis. A full description of these covariates has been provided in a previous paper $^{1}$ and only a summary listing is given here. The factors were selected from the study database on the basis that they were correlated with either the measures of pregnancy history or mental health outcomes and spanned the following domains.

(a) Measures of childhood socioeconomic circumstances including parental education levels, family socioeconomic status, family living standards and family income.

(b) Measures of parental adjustment/family functioning including: number of changes of parent figure during childhood; inter-parental violence; parental adjustment problems (illicit drug use, mental health problems, alcohol problems, criminality); and quality of parental attachments in adolescence.

(c) Measures of child abuse including exposure to childhood sexual or physical abuse prior to age 16 . 
(d) Measures of individual characteristics and educational achievement including measures of neuroticism, self-esteem and novelty seeking in adolescence; childhood conduct problems; child IQ; teacher-rated grade point average.

(e) Measures of adolescent adjustment including early onset sexual intercourse ( $<16$ years), early substance use (tobacco, alcohol and cannabis at age 15), and adolescent mental health problems (depression, anxiety, suicidal ideation).

(f) Time dynamic lifestyle and other factors including: living arrangements in young adulthood (living with parents, cohabitations, age of leaving the family home); and measures of exposure to adverse life events (employment problems, partner relationship problems, serious illness or death in the family, sexual or physical violence victimisation, history of pregnancy loss) in each assessment interval. In addition, to control for the changing history of prior mental health, a lagged measure of the number of mental health problems observed in the previous assessment period was also considered.

\section{Statistical analysis}

Reactions to abortion

Tables 1 and 2 report on the frequency of negative reactions to abortion, positive reactions to abortion, and assessment of the overall abortion decision.

\section{Negative reactions to abortion and mental health}

Table 3 examines the relationship between the number of definite negative reactions to abortion classified into three groups (no problems, one to three problems, four to six problems) and rates of mental health problems (depression, anxiety disorder, suicidal ideation, alcohol dependence, illicit drug dependence and total number of problems) over the period from 15 to 30 years. The estimates in this table were obtained by pooling the data over the four assessment periods (15-18, 18-21, 21-25, 25-30 years) to obtain population averaged estimates of the rates of disorder for each level of abortion-related distress. The association between abortion-related distress and total number of mental health problems was modelled by fitting a repeated measures random effects Poisson regression model to data on: the number of mental health problems reported within a given assessment period (15-18, 18-21, 21-25, 25-30 years); and the number of negative emotional reactions experienced by women who had had an abortion by the end of the assessment period. The fitted model was:

$$
\log \left(Y_{i t}\right)=B_{0 t}+B_{1} X_{i}+v_{i}+e_{i t}
$$

where $\log \left(Y_{i t}\right)$ was the $\log$ rate of mental health problems $(Y)$ for the $i$-th individual in the $t$-th time interval; $X_{\mathrm{i}}$ was the number of negative reactions to abortion reported by the $i$-th participant (scored as: 0 , no distress; 1, one to three problems; 2, four to six problems); $v_{i}$ was an individual specific random effect assumed to be uncorrelated with $X_{i}$; and $e_{i t}$ was the disturbance term for the model. The intercept $B_{0}$ was permitted to vary with time $t$ to allow for changes in the base rate of mental health problems over time. The effect of number of negative reactions to abortion was assumed to be linear with coefficient $B_{1}$. Table 3 reports on the pooled rates of mental health problems for each level of negative reactions and provides estimates of the incidence rate ratio (IRR) and corresponding 95\% CI for each group based on the linear model in Equation 1. The IRR for a one-unit step on the number of negative reactions measure was obtained from the fitted coefficient $B_{1}$ and its standard error in the usual way $e^{B 1 \pm 1.96 s . e .(B 1)}$.

\section{Abortion and subsequent mental health}

The final stage of the analysis examined the relationship between exposure to abortion (classified as: no abortion; abortion with no definite adverse reaction; abortion with one to three definite adverse reactions; abortion with four to six definite adverse reactions) and rates of mental health problems for all female cohort members over the period from 15 to 30 years. This analysis was based on an extension of the analysis reported in a previous paper. ${ }^{1}$ Following this paper two modelling approaches were used.

(a) Concurrent model. In this model the respondent's history of reaction to abortion by the end of a given observation interval was related to mental health outcomes within that interval (15-18, 18-21, 21-25, 25-30 years). The fitted model was:

$$
\begin{array}{r}
\log \left(Y_{i t}\right)=B_{0 t}+B_{1}{ }^{\mathrm{a}} X_{1}{ }_{i t}^{\mathrm{a}}+B_{1}{ }^{\mathrm{b}} X_{1}{ }_{i t}^{\mathrm{b}}+B_{2} X_{2 i t}+ \\
B_{3} X_{3 i t}+B_{4} X_{4 i t}+\Sigma B_{j} Z_{i j}+\Sigma B_{k} Z_{i k t}+v_{i}+e_{i t}
\end{array}
$$

where $X_{1}^{\mathrm{a}}$ it was a time dynamic dichotomous variable reflecting whether the $i$-th participant had an abortion by $t$-th time interval; $X_{1}^{\mathrm{b}}$ it was a continuous variable reflecting the extent of abortion-related distress as in Equation 1 with women who did not have an abortion scored as $0 ; X_{2 i t}, X_{3 i t}$, $X_{4 i t}$ were time dynamic dichotomous measures reflecting exposure of the $i$-th participant to pregnancy loss (miscarriage, stillbirth or ectopic pregnancy), live birth resulting from an unwanted/adverse reaction to pregnancy, or other live birth respectively; $Z_{i j}$ and $Z_{i k t}$ were sets of fixed and time dynamic covariates respectively; $v_{\mathrm{i}}$ was an individual specific random effect and $e_{i t}$ was the disturbance term for the model.

(b) A 5-year lagged model. In this model a strict temporal sequence was imposed on the data so that the assessment of abortion and pregnancy outcomes was restricted to those pregnancies that occurred in the 5-year period prior to the assessment of the mental health outcomes. The specification for this model was similar to the specification of the model in Equation 2 except that the measures of pregnancy/abortion outcomes and time dynamic covariates were lagged in the 5 years preceding the intervals in which mental health outcomes were assessed.

Table 4 shows the IRRs and corresponding 95\% CIs estimated using combinations of the parameters $B_{1}{ }^{\mathrm{a}}, B_{1}{ }^{\mathrm{b}}$ for both models. Estimates of the unadjusted IRRs were also obtained by fitting models that excluded all other predictors from the model except for the measures of abortion. In each case a test of the effect of abortion exposure/abortion-related distress was given by a Wald chi-squared test of the joint null hypothesis $H_{0}: B_{1}{ }^{a}=0 ; B_{1}{ }^{b}=0$. These analyses were restricted to an examination of the total number of mental health problems reported in each interval since attempts to analyse individual diagnoses (depression, anxiety disorder, suicidal ideation, alcohol dependence, illicit drug dependence) proved to be unsatisfactory owing to problems relating to sparse cell sizes, inflated standard errors and unstable parameter estimates. For these reasons no clear conclusions can be drawn about the links between reactions to abortion and specific mental health outcomes. All models were fitted using Stata version 10 for Windows.

\section{Sample sizes}

The analysis of initial reactions to abortion was based on a sample of 104 women who had experienced an induced abortion and who 
were interviewed at age 30 . This sample represented $89 \%$ of all those women $(n=117)$ reporting an abortion by age 30 . The analysis of the associations between abortion/pregnancy history and rates of mental health problems was based on a sample of 532 women with data available on abortion history and mental health outcomes for at least one assessment period from 15 to 30 years. This sample represented $84 \%$ of the initial cohort of 630 females. As described in a previous paper, ${ }^{1}$ data weighting methods were used to examine the extent to which the pattern of missing data may have influenced study conclusions. This analysis produced almost identical conclusions to the results reported here, suggesting that the findings were unlikely to have been influenced by selection bias.

\section{Results}

\section{Reported reactions to abortion}

Tables 1 and 2 describe women's reaction to abortion as reported at age 30. Table 1 reports on negative and positive reactions to abortion, and Table 2 reports on the woman's overall assessment at age 30 of the appropriateness of the abortion decision.

In Table 1, the section on negative reactions suggests that the majority of women reported at least one negative emotional reaction, including: sorrow, sadness, guilt, regret, grief, and disappointment. Over $85 \%$ of the group mentioned at least one of these, with $34.6 \%$ reporting five or more negative reactions. In terms of definite reactions to the abortion in which the woman reported that she had experienced the reaction 'very much', over $55 \%$ of the sample reported at least one definite reaction, with $34.6 \%$ reporting thee or more such reactions. These results clearly suggest that there was a spectrum of negative reaction ranging from a minority of women who reported no negative response, to a group who reported several definite negative reactions.

In part, the high frequency of negative reactions was offset by a number of positive reactions, including: relief, happiness, and satisfaction. Over $86 \%$ of women reported at least one positive reaction, with $29.8 \%$ reporting all three reactions. In terms of

Table 1 Women reporting negative and positive reactions to first abortion, ages $15-30(n=104)$

\begin{tabular}{|lccc|} 
& \multicolumn{3}{c|}{ Response, \% reporting } \\
\cline { 2 - 4 } & Not at all & Somewhat & Very much \\
Negative reactions & & 37.5 & \\
$\quad$ Sorrow & 31.7 & 36.5 & 30.8 \\
Sadness & 28.9 & 29.8 & 33.7 \\
Guilt & 36.5 & 18.3 & 14.4 \\
Regret & 67.3 & 25.0 & 23.1 \\
Grief/loss & 51.9 & 28.9 & 32.7 \\
Disappointment & 38.5 & 30.8 & \\
\hline Positive reaction & & 19.2 & 23.1 \\
Relief & 15.4 & 19.2 & 20.2 \\
Happiness & 57.7 & \\
Satisfaction & 60.6 & & \\
\hline
\end{tabular}

Table 2 Overall evaluation of decision of women reporting first abortion, ages $15-30(n=104)$

\begin{tabular}{ccc}
$\begin{array}{c}\text { Definitely } \\
\text { right } \\
\text { decision }\end{array}$ & $\begin{array}{c}\text { Not } \\
\text { sure }\end{array}$ & $\begin{array}{c}\text { Definitely } \\
\text { wrong } \\
\text { decision }\end{array}$ \\
89.4 & 8.7 & 1.9 \\
\hline
\end{tabular}

definite reactions, $59.6 \%$ reported one or more definite reactions, with $12.5 \%$ reporting three definite positive reactions.

The findings suggest that many women experienced a mixture of both positive and negative emotions about having an abortion. These findings raise the issue of the extent to which the decision to have an abortion was seen to be correct. As may be seen from Table 2, nearly $90 \%$ of women questioned at age 30 thought the decision was correct, and only $2 \%$ believed it to be incorrect.

In general, the findings in Tables 1 and 2 suggest that although women experienced a mix of positive and negative emotions about having an abortion, an overwhelming majority reported that they believed they had made the right decision in having an abortion.

\section{Negative reactions to abortion and subsequent mental health}

The findings in Tables 1 and 2 raise the important issue of the extent to which reactions to abortion were prognostic of mental health outcomes after abortion. Table 3 shows the associations between the extent of distress reported by the women subsequent to abortion and rates of mental health problems (depression, anxiety, suicidal ideation, alcohol dependence, illicit drug dependence, total number of mental health problems). Distress was measured using a count of the number of items in Table 1 to which the women made a definite negative response, classified into three levels (no negative reactions, one to three negative reactions, four to six negative reactions). As explained in the Method, rates of mental health problems have been pooled over all observation periods (ages 15-18, 18-21, 21-25, 25-30 years) to produce population-averaged rate estimates for each outcome, and rates have been reported using both concurrent and 5-year lagged models for the assessment of the timing of abortion. Table 3 also reports the estimated IRR and corresponding 95\% CI for the total number of mental health problems for each level of distress relative to those who reported no adverse reactions to abortion.

For the concurrent model, increasing abortion-related distress was associated with increasing rates of mental health problems. These trends were most evident for major depression, anxiety disorder and suicidal behaviour, and least evident for alcohol dependence and illicit drug dependence. A random effects model fitted to the overall rates of mental health problems showed a significant linear association $(P=0.04)$ with the extent of reported distress. Overall, women reporting four or more distress responses had rates of mental health problems that were approximately 1.5 times higher than those of women not reporting distress.

The 5-year lagged model showed that risks of all disorders tended to increase with increasing symptoms of distress. A random effects model fitted to the overall rates of mental health problems also showed a significant linear association $(P=0.047)$ with the extent of reported distress. Overall, women reporting four or more distress responses had rates of mental health problems that were also 1.5 times higher than for women exposed to abortion who did not report distress.

A parallel analysis using the number of positive responses to abortion failed to show any association between the number of positive responses and rates of mental health problems. The findings in Table 3 clearly suggest that increasing distress associated with abortion was associated with increased risks of mental health problems.

\section{Distress as a moderating factor}

A previous paper $^{1}$ showed that even following extensive control for confounding factors, exposure to abortion was associated with 


\begin{tabular}{|c|c|c|c|}
\hline \multirow[b]{2}{*}{ Measure } & \multicolumn{3}{|c|}{ Definite negative reactions to abortion, $n$} \\
\hline & 0 & $1-3$ & $4-6$ \\
\hline \multicolumn{4}{|l|}{ Concurrent models ${ }^{\mathrm{a}}$} \\
\hline Major depression, \% & 32.9 & 39.4 & 58.3 \\
\hline Anxiety disorder, \% & 30.1 & 37.4 & 37.5 \\
\hline Suicidal ideation, \% & 13.7 & 22.2 & 29.2 \\
\hline Alcohol dependence, \% & 6.2 & 4.0 & 6.3 \\
\hline Illicit drug dependence, \% & 10.3 & 10.1 & 8.3 \\
\hline Number of mental health problems, mean & 0.93 & 1.13 & 1.40 \\
\hline IRR $(95 \% \mathrm{Cl})$ & 1 & $1.25(1.01-1.55)$ & $1.56(1.02-2.40)$ \\
\hline \multicolumn{4}{|l|}{ 5-year lagged models ${ }^{\mathrm{b}}$} \\
\hline Major depression, \% & 28.0 & 41.0 & 61.1 \\
\hline Anxiety disorder, \% & 37.3 & 41.0 & 38.9 \\
\hline Suicidal ideation, \% & 14.7 & 23.1 & 38.9 \\
\hline Alcohol dependence, \% & 9.3 & 5.1 & 11.1 \\
\hline Illicit drug dependence, \% & 9.3 & 12.8 & 16.7 \\
\hline Number of mental health problems, mean & 0.99 & 1.23 & 1.67 \\
\hline IRR $(95 \% \mathrm{Cl})$ & 1 & $1.23(1.00-1.51)$ & $1.51(1.01-2.27)$ \\
\hline
\end{tabular}

moderate increases in rates of mental health problems (with IRRs of approximately 1.3). The findings in Table 3 raise the clear possibility that the association between abortion and mental health outcomes may be modified by the degree of distress associated with the abortion. To examine this issue, the previous analysis of the association between overall rates of mental health problems and abortion was extended to incorporate the extent of reported distress associated with abortion by classifying those having an abortion into the three groups shown in Table 3: those reporting no definite adverse response; those reporting one to three definite negative reactions; and those reporting four to six definite negative reactions. The results of this re-analysis are presented in Table 4, which summarises the results for the two modelling methods - concurrent and 5-year lagged analysis (see Method for an account of these models). These models were based on data for 532 women observed during the period 15-30 years.

For each model, Table 4 reports: the unadjusted associations between abortion history (subdivided by degree of abortionrelated distress) and overall rates of mental health problems; and the covariate adjusted associations. In each case, the associations are summarised by the IRRs and corresponding 95\% CIs for the rate of mental health problems for each level of abortion-related distress relative to those not exposed to abortion. The adjusted analysis considered a wide range of covariates based on our previous analysis. ${ }^{1}$ These covariates included measures of: childhood socioeconomic circumstances; childhood family functioning; parental adjustment; exposure to abuse in childhood; individual characteristics; educational achievement; adolescent adjustment; other pregnancy outcomes (e.g. pregnancy loss, live birth); and other time dynamic lifestyle and related factors (see Method). Table 4 also lists the significant covariates for each model.

\section{Unadjusted results}

For both models there was clear evidence of a relationship in which rates of mental health problems increased significantly $(P<0.001)$ with increasing abortion-related distress. Both models suggest that those having abortions but not reporting definite distress had rates of mental health problems that were approximately 1.3 times higher than for those not having an abortion; those having an abortion and reporting one to three definite negative reactions had rates that were approximately 1.6 times higher; and those having an abortion and reporting four to six definite negative reactions had rates that were approximately 1.9-2.0 times higher than for those not having an abortion. For the concurrent model, rates of mental health problems were significantly higher at all levels of distress than for those not having an abortion, whereas for the 5-year lagged model rates were significantly higher only among those who reported definite negative reactions to abortion.

\section{Table 4 Associations between abortion history (subdivided by degree of abortion-related distress) and rates of mental health} problems (15-30 years) before and after adjustment for covariates $(n=532)$

\begin{tabular}{|c|c|c|c|c|c|}
\hline Measure & $\begin{array}{c}\text { No } \\
\text { abortion }\end{array}$ & $\begin{array}{l}\text { Abortion with no } \\
\text { negative reactions }\end{array}$ & $\begin{array}{l}\text { Abortion with } 1-3 \\
\text { negative reactions }\end{array}$ & $\begin{array}{l}\text { Abortion with } 4-6 \\
\text { negative reactions }\end{array}$ & $\begin{array}{l}\text { Significant } \\
\text { covariates }^{\mathrm{a}}\end{array}$ \\
\hline \multicolumn{6}{|l|}{ Concurrent model } \\
\hline Unadjusted IRR (95\% Cl) & $1(-)$ & $1.33 *(1.04-1.70)$ & $1.62^{* * *}(1.33-1.98)$ & $1.99 * * *(1.40-2.79)$ & \\
\hline Adjusted IRR (95\% Cl) & $1(-)$ & $1.24(0.99-1.55)$ & $1.43^{* * *}(1.20-1.70)$ & $1.64^{\star \star}(1.23-2.20)$ & $1-11$ \\
\hline \multicolumn{6}{|l|}{ 5-year lagged model } \\
\hline Unadjusted IRR (95\% Cl) & $1(-)$ & $1.29(0.96-1.72)$ & $1.58^{* * *}(1.24-2.01)$ & $1.94 * * *(1.29-2.91)$ & \\
\hline Adjusted IRR (95\% Cl) & $1(-)$ & $1.14(0.84-1.53)$ & $1.43^{* *}(1.12-1.83)$ & $1.81 * * \quad(1.19-2.75)$ & $1-5,8,9$ \\
\hline \multicolumn{6}{|c|}{$\begin{array}{l}\text { IRR, incidence rate ratio. } \\
\text { a. Significant covariates: } 1 \text {, childhood sexual abuse; } 2 \text {, childhood physical abuse; } 3 \text {, neuroticism (14 years); } 4 \text {, self-esteem }(15 \text { years); } 5 \text {, teacher-rated grade point average } \\
\text { (11-13 years); } 6 \text {, cohabiting partner; } 7 \text {, partner relationship problems; } 8 \text {, serious illness or death in family; } 9 \text {, sexual or physical violence victimisation; } 10 \text {, lagged history of mental } \\
\text { health problems; } 11 \text {, history of pregnancy loss. } \\
{ }^{*} P<0.05, * * P<0.01, * * * P<0.001 \text {. }\end{array}$} \\
\hline
\end{tabular}




\section{Adjusted results}

For both models, adjustment for confounding factors tended to reduce the size of the associations but clear and significant $(P<0.001)$ relationships remained between the extent of distress associated with abortion and rates of mental health problems. Specifically, after adjustment for covariates the association between abortion with no negative reactions and mental health problems became non-significant for both models, with risk ratios ranging from 1.14 to 1.24 ; whereas those reporting distress had significantly higher rates of mental health problems, with these rates increasing with the extent of distress. The net effect of these trends was that those exposed to abortion and reporting four or more definite negative reactions had adjusted rates of mental health problems that were between 1.64 and 1.81 times higher than for those not exposed to abortion $(P<0.01)$.

More generally, the results from both models suggested that the relationship between unwanted pregnancy terminated by abortion and mental health depended critically on the extent to which abortion was associated with distress and that any increase in risks of mental health problems in women exposed to abortion was largely confined to those women who reported one or more definite symptoms of distress.

\section{Discussion}

In this study we have examined the extent to which the association between abortion and mental health found in a previous study ${ }^{1}$ was modified by the woman's reactions to the abortion. The key findings of this study are summarised below.

\section{Reactions to abortion}

In agreement with previous research in this area, ${ }^{4-14}$ women reported a range of reactions to abortion with a substantial number reporting feelings of grief, guilt, loss and related emotions in response to abortion. Over $85 \%$ of women reported at least one of these adverse reactions, with a third reporting five or more of these reactions. However, these negative reactions to abortion were offset by positive responses including relief, happiness and satisfaction, with these responses being noted by over $85 \%$ of women. Importantly, on the basis of assessments made at age 30 , nearly $90 \%$ stated that the decision to have an abortion was the correct one, and only $2 \%$ reported that they believed the decision to be incorrect. These findings are consistent with previous research which suggests that the great majority of women do not regret the decision to have an abortion, ${ }^{4,6}$ and the accumulated evidence on this topic does not support recent claims from pro-life advocates that large numbers of women who have abortions regret their decision. ${ }^{18}$

\section{Adverse reactions and mental health outcomes}

The second stage of the analysis examined the relationship between the extent of adverse reaction to the abortion and risks of subsequent mental health outcomes. This analysis suggested the presence of a clear relationship in which risks of subsequent mental health problems increased with the extent of distress, upset or guilt reported by the woman: women reporting four or more negative reactions had rates of subsequent mental health problems that were 1.5 times higher than women reporting no distress.

These findings were then incorporated into general multivariate models aimed at assessing the relationship between the extent of distress to abortion and subsequent mental health taking into account a wide range of covariate factors. These analyses confirmed the general conclusions that increasing reports of abortion-related distress were associated with increasing risks of mental disorders: women who reported at least one negative reaction to the abortion had rates of mental health problems that were approximately 1.4-1.8 times higher than women not exposed to abortion, and between 1.2 and 1.6 times higher than women who were exposed to abortion but did not report any adverse reactions to abortion. All of these findings are consistent with the conclusion that unwanted pregnancy terminated by abortion is an adverse life event that increases risks of mental health problems, with these increases in risk being proportional to the degree of distress associated with the abortion of an unwanted pregnancy.

The finding that the extent of distress associated with abortion modifies the risks of subsequent mental health problems is consistent with the view that it is the woman's reaction to abortion that increases risks of mental health rather than the experience of unwanted pregnancy. Our results suggest that women who have unwanted pregnancy terminated by abortion who do not experience distress do not show increased risks of mental health problems. This finding is consistent with the results of our previous study that showed that unwanted pregnancies that came to term were not associated with a detectable increase in risks of mental health problems. ${ }^{1}$

Combining the findings of our two studies leads to the following generalisations about the links between unwanted pregnancy, abortion and mental health in this birth cohort.

(a) First, unwanted pregnancy terminated by abortion was associated with modestly increased risks of common mental health problems for women who reported significant distress about the abortion $(\mathrm{RR}=1.4-1.8)$.

(b) Second, unwanted pregnancy terminated by abortion was not associated with significantly increased risks of mental health problems for women who did not report significant distress about the abortion $(\mathrm{RR}=1.14-1.24)$.

(c) Third, unwanted pregnancy that came to term was not associated with significant increases in mental health problems $(\mathrm{RR}=1.05-1.11)$.

(d) Finally, any associations between unwanted pregnancy, abortion and mental health problems were small to moderate, with adjusted relative risks in the region of 1.1-1.8. Estimates of the population attributable risk suggested that exposure to unwanted pregnancy terminated by abortion accounted for fewer than $5 \%$ of the mental health problems experienced by women in this cohort. ${ }^{1}$

As we have noted previously, ${ }^{1}$ these findings are not consistent with strong pro-life positions that depict unwanted pregnancy terminated by abortion as having devastating consequences for women's mental health. ${ }^{19}$ Equally, however, the findings do not support strong pro-choice positions that claim unwanted pregnancy terminated by abortion is without mental health risks. ${ }^{20}$ Rather, the accumulated evidence suggests that unwanted pregnancy terminated by abortion is an event that leads to significant distress in some women, with this distress being associated with a modest increase in risk of common mental health problems.

\section{Limitations}

These conclusions need to be interpreted in the light of a number of potential limitations of the study. The greatest limitation is that the assessment of abortion-related distress was based on retrospective reports obtained at the age of 30 . Such reports may be subject to errors of reminiscence and possible recall bias. For this 
reason it is important that our findings are replicated using prospective data to assess the links between abortion distress and subsequent mental health. Although a number of studies have reported on rates of abortion distress, to date no study appears to have linked this distress to later mental health outcomes, with the possible exception of Major et al who reported correlations of $0.25-0.40$ between the extent of abortion-related distress and subsequent mental health problems. ${ }^{4}$ That study is consistent with the view that the extent of distress associated with abortion plays a significant role in determining subsequent mental health outcomes. It addition, as is the case with all observational studies, conclusions may be influenced by omitted confounders and errors of measurement in the assessments of exposure and outcome. Further, the present data are unclear as to whether negative reactions to abortion were more common among participants who were members of communities or groups in which an unwanted pregnancy or abortion may have been viewed most negatively. Nonetheless, the accumulated evidence is consistent with the major conclusions outlined above.

\section{Implications}

These conclusions have implications for both service provision and the interpretation of the law in jurisdictions such as New Zealand, and England and Wales. The finding that the extent of distress caused by the abortion is a predictor of subsequent mental health suggests the need for providers of abortion to: conduct thorough screening of abortion-related distress; to carry out adequate follow-up of those showing distress; and to counsel those showing distress about future mental health risks and the need for support. ${ }^{10,21}$ In terms of legal issues, our findings have important implications for jurisdictions such as England and Wales, and New Zealand where over $90 \%$ of abortions are authorised on the grounds that proceeding with the unwanted pregnancy would pose a serious threat to the woman's mental health. ${ }^{22,23}$ There is no evidence in this research that would suggest that unwanted pregnancies that come to term were associated with increased risks of mental health problems or that abortion mitigated the risks of mental health problems in women having unwanted pregnancy.

In addition, although recent reviews of the evidence ${ }^{2,3}$ have concluded that abortion is not associated with increased mental health risks when compared with unwanted pregnancies that come to term, no review to date has found that abortion is associated with a reduction in mental health risks. Collectively, this evidence raises important questions about the practice of justifying termination of pregnancy on the grounds that this procedure will reduce risks of mental health problems in women having an unwanted pregnancy. Currently there is no evidence to support the assumptions underlying this practice, and the findings of the present study suggest that abortion may, in fact, increase mental health risks among those women who find seeking and obtaining an abortion a distressing experience.

\footnotetext{
David M. Fergusson, PhD, L. John Horwood, MSC, Joseph M. Boden, PhD,

David M. Fergusson, PhD, L. John Horwood, MSC, Joseph M. Boden, PhD,
Christchurch Health and Development Study, Department of Psychological Medicine, University of Otago, Christchurch School of Medicine and Health Sciences, Christchurch, New Zealand

Correspondence: David M. Fergusson, Christchurch Health and Development Study, University of Otago, Christchurch School of Medicine and Health Sciences, PO Box 4345, Christchurch, New Zealand. Email: dm.fergusson@otago.ac.nz

First received 11 Mar 2009, final revision 23 Apr 2009, accepted 6 May 2009
}

\section{Funding}

This research was funded by grants from the Health Research Council of New Zealand, the National Child Health Research Foundation, the Canterbury Medical Research Foundation and the New Zealand Lottery Grants Board.

\section{References}

1 Fergusson DM, Horwood L, Boden JM. Abortion and mental health disorders: evidence from a 30-year longitudinal study. Br J Psychiatry 2008; 193: 444-51.

2 Charles VE, Polis CB, Sridhara SK, Blum RW. Abortion and long-term mental health outcomes: a systematic review of the evidence. Contraception 2008; 78: $436-50$

3 American Psychological Association. Report of the APA Task Force on Mental Health and Abortion. APA, 2008.

4 Major B, Cozzarelli C, Cooper ML, Zubek J, Richards C, Wilhite M, et al. Psychological responses of women after first-trimester abortion. Arch Gen Psychiatry 2000; 57: 777-84.

5 Broen AN, Moum T, Bodtker AS, Ekeberg O. Psychological impact on women of miscarriage versus induced abortion: a 2-year follow-up study. Psychosom Med 2004; 66: 265-71.

6 Quinton WJ, Major B, Richards C. Adolescents and adjustment to abortion: are minors at greater risk? Psychol Public Policy Law 2001; 7: 491-514.

7 Kero A, Hogberg U, Lalos A. Wellbeing and mental growth-long-term effects of legal abortion. Soc Sci Med. 2004; 58: 2559-69.

8 Major B, Richards C, Cooper M, Cozzarelli C, Zubek J. Personal resilience, cognitive appraisals, and coping: an integrative model of adjustment to abortion. J Pers Soc Psychol 1998; 74: 735-52.

9 Major B, Mueller P, Hildebrandt K. Attributions, expectations, and coping with abortion. J Pers Soc Psychol 1985; 48: 585-99.

10 Mueller $P$, Major B. Self-blame, self-efficacy, and adjustment to abortion. J Pers Soc Psychol 1989; 57: 1059-68.

11 Major B, Cozzarelli C, Sciacchitano AM, Cooper M, Testa M, Mueller PM. Perceived social support, self-efficacy, and adjustment to abortion. J Pers Soc Psychol 1990; 59: 452-63.

12 Pope LM, Adler NE, Tschann J. Postabortion psychological adjustment: are minors at increased risk? J Adolesc Health 2001; 29: 2-11.

13 Soderberg H, Janzon L, Sjoberg NO. Emotional distress following induced abortion: a study of its incidence and determinants among abortees in Malmo, Sweden. Eur J Obstet Gynecol Reprod Biol 1998; 79: $173-8$.

14 Kero A, Lalos A. Ambivalence - a logical response to legal abortion: a prospective study among women and men. J Psychosom Obstet Gynaecol 2000; 21: 81-91.

15 Costello A, Edelbrock C, Kalas R, Kessler M, Klaric SA. Diagnostic Interview Schedule for Children (DISC). National Institute of Mental Health, 1982.

16 World Health Organization. Composite International Diagnostic Interview (CIDI). WHO, 1993.

17 American Psychiatric Association. Diagnostic and Statistical Manual of Mental Disorders, (4th edn) (DSM-IV). APA, 1994

18 Burke T, Reardon DC. Forbidden Grief: The Unspoken Pain of Abortion. Acorn Books, 2002.

19 Agee MC. The Health Risks of Abortion. Family Research Council 2003 (http:// heartland.temp.siteexecutive.com/pdf/32201f.pdf).

20 Planned Parenthood Federation of America. Abortion. Planned Parenthood Federation of America, 2006 (http://www.plannedparenthood.org/healthtopics/abortion-4260.htm).

21 Needle RB, Walker LEA. Abortion Counselling. Springer, 2007.

22 Statistics New Zealand. Abortion Statistics: Year Ended December 2004. Statistics New Zealand, 2005 (http://www.stats.govt.nz/browse_for_stats/ health/abortion/abortionstatistics_hotpdec04.aspx).

23 Department of Health. Abortion Statistics, England and Wales: 2003. Statistical Bulletin 2004/14. Department of Health, 2004. 